\title{
La tutoría como estrategia de trabajo compartido
}

\author{
Tutoring as a shared work strategy
}

\author{
Carlota de León Huertas ${ }^{1}$, Ignacio González López² \& Mª Dolores Eslava \\ Suanes $^{3}$
}

Fecha de recepción: 01/10/2018; Fecha de revisión: 25/02/2019; Fecha de aceptación: 24/05/2019

Cómo citar este artículo:

León Huertas, C. de León, González López, I. \& Eslava Suanes, M.D. (2019). Título del artículo en español. Revista de Innovación y Buenas Prácticas Docentes, 8(2), 23-33.

Autor de Correspondencia: ignacio.gonzalez@uco.es

\section{Resumen:}

Se entiende la labor tutorial como una parte esencial de la responsabilidad de la docencia universitaria en la que se busca establecer una interacción más personalizada entre el equipo docente, los y las agentes sociales y el alumnado mentor con los y las estudiantes, lo que supone adaptar el aprendizaje a sus condiciones y necesidades, tanto individuales como grupales, de tal manera que cada alumno y alumna logre alcanzar las competencias requeridas para asumir un proyecto de trabajo a realizar en la titulación de Grado de Educación Social. El objetivo general que centra el desarrollo de esta acción innovadora se concreta en detectar las necesidades de acción tutorial del alumnado de Educación Social en el abordaje de un proyecto de trabajo coordinado en el marco de las materias "Animación Sociocultural: proyectos e intervención", "Métodos de Investigación en Educación Social", de la titulación de Grado Educación Social. Nuestro propósito es sentar las bases para la elaboración de un plan de acción tutorial universitaria que agrupe las acciones de acompañamiento y seguimiento del profesorado universitario responsable de las materias implicadas, del alumnado mentor y de los y las agentes externas adheridos al proyecto.

Palabras clave: asesoramiento, intervención socioeducativa, mentoría, trabajo colaborativo.

\section{Abstract:}

The tutorial work is an essential part of the responsibility of university teaching in which it seeks to establish a more personalized interaction between the teaching team, social agents and mentors with students, which means adapting the learning to their conditions and needs, both individual and group, so that each student manages to achieve the skills required to take on a project of work to be carried out in the Degree of Social Education. The general objective that focuses the development of this innovative action is concrete in detecting the needs of tutorial action of the students of Social Education in the approach of a project of work coordinated in the framework of the subjects "Sociocultural Animation: projects and intervention", "Methods of Research in Social Education" of the Degree in Social Education. Our purpose is to lay the foundations for the elaboration of a university tutorial action plan that groups together the actions of accompaniment and follow-up of university professors responsible for the matters involved, of the mentor student and of the external agents attached to the project

Key Words: advice, socio-educational intervention, mentoring, collaborative work.

\footnotetext{
1 Universidad de Córdoba (España), carlota.leon.huertas@uco.es; CÓDIGO ORCID: 0000-0002-71258637

2 Universidad de Córdoba (España), ignacio.gonzalez@uco.es; CÓDIGO ORCID: 0000-0002-8215-2563

3 Universidad de Córdoba (España), m62essum@uco.es; CÓDIGO ORCID: 0000-0002-7148-7106
} 


\section{INTRODUCCIÓN}

Las enseñanzas conducentes al título de Educación Social están orientadas a proporcionar una formación científica y experiencial adecuada en los campos de la educación no formal: educación de adultos, inserción social de personas desadaptadas y con discapacidad y en la acción socioeducativa propiamente dicha. En el marco de esos ámbitos, el educador o educadora social es un profesional que trata de dar respuesta a demandas y necesidades sociales, previniendo, paliando o corrigiendo procesos de exclusión social, al tiempo que promueve también procesos de inclusión y participación social (Ruiz Corbella, Martín Cuadrado \& Cano Ramos, 2015). Para ello, diseña, gestiona, desarrolla y evalúa planes, programas, proyectos y acciones educativas adaptadas a los contextos en los que se inscribe su actuación con el fin de que logren su desarrollo, su integración y participación en la comunidad en diferentes ámbitos (social, político, económico y cultural).

La Universidad por la que se apuesta debe formar ciudadanía crítica, responsable y productiva socialmente, de forma que interiorice el compromiso de su participación en los procesos de transformación social. Y es en este marco en el que integramos este trabajo, combinando los contenidos teóricos de las asignaturas de "Animación Sociocultural: proyectos e intervención" y "Métodos de Investigación en Educación Social" del segundo curso del Grado en Educación Social de la Universidad de Córdoba e invitando a nuestro alumnado a conocer, analizar y hacer propuestas inclusivas enmarcadas en un proyecto académico de investigación e intervención en contextos inclusivos. Para dar respuesta a los objetivos del trabajo, las y los estudiantes se incorporan a un escenario de trabajo previamente negociado con el tejido social en el cuál se implementará la investigación y se proyectará la intervención.

La presencia en estos entornos está apoyada por la existencia de la figura del tutor y/o tutora externa, referentes de las entidades sociales con las que se colabora en los proyectos, cuyo objetivo fundamental es establecer una coordinación periódica para el seguimiento y evaluación de las investigaciones con el profesorado de las dos materias implicadas, ofrecer recursos espacio-temporales para el trabajo y coordinar y asesorar al grupo de trabajo de estudiantes en el contexto del centro de acogida.

Así mismo, se reclama la figura del mentor o mentora que facilita al alumnado en curso la ayuda necesaria para abordar con éxito las demandas del proyecto. En este caso, las y los mentores son los y las compañeras del curso superior que ya han participado en la experiencia y que aceptan participar en el programa para lo cual recibirán formación como mentores, potenciando en ellos y en ellas habilidades sociales, de relación, orientación y liderazgo (Fernández-Salinero, 2014).

El proceso de conciliación de estas dos asignaturas va a requerir entender la tutoría como un elemento facilitador y dinamizador de la acción docente. De este modo, y como apunta Álvarez González (2008), el acompañamiento en el proceso de enseñanza-aprendizaje del alumnado proporciona información que, a la par que optimiza la práctica docente, apunta modos de trabajo que nos permiten detectar las necesidades formativas del alumnado para afrontar el proyecto planteado.

Se enciente la tarea tutorial como una parte esencial de la responsabilidad de la docencia en la que se busca establecer una interacción más personalizada entre el equipo docente -asumiendo que el equipo docente está formado por el profesorado de la universidad, los agentes responsables de cada institución y el grupo mentor- y el alumnado, adaptando, de este modo, el aprendizaje a sus condiciones y necesidades, tanto individuales como grupales, de tal manera que cada estudiante logre alcanzar las competencias requeridas para asumir un proyecto de investigación e intervención en contextos de inclusión cuando se incorpore al escenario laboral. 


\section{OBJETIVOS}

La finalidad general del trabajo es establecer relaciones significativas entre los conocimientos conceptuales de dos asignaturas del segundo curso del Grado en Educación social (Métodos de Investigación en Educación Social y Animación Sociocultural: proyectos e intervención) y diferentes formas y estrategias de intervención socioeducativa, mediante la experimentación de estrategias y técnicas que posibiliten la implicación sustantiva del alumnado en su proceso formativo con el fin de aproximar su formación universitaria a la realidad profesional en la que ejercerán su trabajo. Ello implica una reflexión sobre las características de un proceso de intervención socioeducativa en contextos no formales y los diferentes instrumentos que se usan habitualmente en este proceso.

Asimismo, otros objetivos que se espera conseguir mediante el desarrollo de estas prácticas co-tuteladas son:

- Detectar las necesidades de asesoramiento del alumnado en la dimensión personal de la formación: habilidades para debatir en clase, argumentar y hablar en público, conocimiento del tejido asociativo de la ciudad de Córdoba y participación activa en redes de colaboración ciudadana.

- Detectar las necesidades de asesoramiento del alumnado en la dimensión académica de la formación: toma de decisiones académicas, adaptación a la metodología de las materias, capacidad para trabajar en equipo, exposición oral de resultados académicos, información sobre recursos relacionados con las materias (informáticos, formación continua, bibliográficos, etc.) y conocimiento de las instituciones del entorno relacionadas con la formación en atención a la diversidad.

- Detectar las necesidades de asesoramiento del alumnado en la dimensión profesional de la formación: desarrollo de competencias profesionales, habilidades para la empleabilidad, conocimiento de las salidas laborales acordes con el proyecto de trabajo realizado y fomento de la colaboración con las agencias del entorno social.

- Para responder a esta meta se han diseñado unas prácticas integradas con el fin de que los alumnos y las alumnas puedan utilizar los conocimientos que van adquiriendo en las asignaturas implicada, con el fin de analizar y valorar algunos de los referentes que condicionan el trabajo de intervención de la Educación Social.

Se ha invitado a participar en esta propuesta de aula al tejido asociativo de la ciudad, así como a cinco estudiantes al amparo de la figura de alumnado mentor. Es por ello que es intención de este trabajo generar una cultura colaborativa entre el profesorado participante, el tejido asociativo y las y los mentores para dar una mejor respuesta a las demandas del alumnado en el ejercicio de su futura profesión.

\section{DESARROLLO DE LA EXPERIENCIA DE INNOVACIÓN}

Este trabajo se ha realizado en tres fases diferenciadas, pero íntimamente conectadas y que tratan de resolver los objetivos anteriormente formulados, entendiéndose el método empleado como mutifásico secuencial (Tashakkori \& Teddlie, 2003): 
Fase 1: Integración de créditos prácticos para la formación en competencias de intervención socioeducativa: en primer lugar, se establecieron las coordenadas para la puesta en marcha del procedimiento de integración conjunta de los créditos prácticos de las materias implicadas por parte del profesorado implicado: diseño de las actividades prácticas que deberán llevar a cabo los alumnos y alumnas en el entorno de las asignaturas implicadas (elaboración de mapas de relaciones a partir de la investigaciones en escenarios reales de intervención socioeducativa). Las prácticas de ambas asignaturas contaron con la presencia del profesorado implicado en las dos materias, de modo que se realizó una integración real de estas actividades (González López \& León Huertas, 2015). Durante el transcurso del periodo docente de impartición de las materias, el alumnado del segundo curso del Grado en Educación Social diseñó e implementó un proyecto de investigación e intervención basado en la generación de mapas de relaciones (evocación de las interacciones existentes entre un grupo meta o sujeto de estudio y una serie de grupos periféricos en escenarios reales de carácter socioeducativo no formal) y el diseño de un plan de intervención a partir de los resultados obtenidos. El objeto de este plan es facilitar la creación de entornos inclusivos (Arnáiz Sánchez, Martínez Abellán \& López Castaño, 2000). Se parte de un estudio de necesidades que son evocadas por un grupo meta (objeto de intervención socioeducativa) a partir de un diagnóstico o evaluación inicial siguiendo procedimientos derivados de la investigación evaluativa, en referencia a una serie de grupos periféricos o elementos contextuales que inciden sobre esa necesidad. Seguidamente, y con los resultados obtenidos, el alumnado establece las relaciones que existen entre todos los elementos que condicionan el proyecto, partiendo del grupo meta hacia los grupos periféricos y viceversa, o entre los grupos periféricos entre sí. Dichas relaciones (en las que se especifica su tipología, dirección e intensidad) tienen un doble carácter, las que favorecen la generación de entornos inclusivos y las que obstaculizan dicho objetivo. Finalmente, partiendo de esa estructura relacional final, el alumnado promueve y desarrolla una serie de propuestas de intervención para mantener las relaciones que facilitan el entorno inclusivo y transformar las relaciones que obstaculizan el entorno inclusivo. Por medio del uso de tutorías en gran grupo presenciales en el aula, tutorías en pequeños grupos en el aula y en el despacho del profesorado, así como tutorías virtuales, el alumnado pondrá en práctica las tareas propuestas. La finalización de la docencia en las asignaturas implicadas precisó de la puesta en marcha de un cuestionario de valoración final del proceso de integración de los créditos prácticos de las asignaturas implicadas en este proyecto.

Fase 2: Detección de las necesidades de asesoramiento del alumnado: en esta segunda fase se ha buscado, con la ayuda de un cuestionario de elaboración propia, recoger información sobre las necesidades de asesoramiento en las dimensiones personal, académica y profesional del alumnado matriculado en el segundo curso del Grado en Educación Social, con el fin de identificar las principales demandas que permitan consolidar, en posteriores cursos académicos, un plan de acompañamiento tutorial en el desarrollo de las actividades emanadas en la fase 1 de este trabajo.

Fase 3: Generación de una cultura colaborativa entre el profesorado, las agencias sociales y el alumnado mentor: se generó un espacio de trabajo y reflexión conjunta del equipo docente implicado en este proyecto al que se invitó a los y las agentes sociales (Fundación Cruz Blanca y Mujeres en Zona de Conflicto) y al alumnado mentor (tres alumnas y dos alumnos de $4^{\circ}$ curso del Grado en Educación Social), en forma de sesiones de trabajo quincenal, que respondieron a la dinámica de apoyo mutuo donde se trabajaron, entre otros, los siguientes temas: a) aplicación práctica de los contenidos impartidos en las sesiones teóricas, b) metodologías de aula, c) formas de evaluación del alumnado y d) técnicas, instrumentos y recursos de apoyo a las actividades docentes. Por otro lado, se impulsaron acciones formativas para facilitar el acompañamiento de la mentoría en las sesiones de aula dedicadas a las prácticas anteriormente descritas, en las dimensiones que aquí se citan: habilidades sociales, 
escucha activa, liderazgo transformacional, resolución de conflictos, toma de decisiones y gestión eficaz del tiempo.

\section{RESULTADOS}

Del total de estudiantes matriculados en las materias implicadas en esta propuesta práctica $(\mathrm{N}=64)$, hemos obtenido evidencias sobre la valoración de la misma de un total de 56 estudiantes (87.50\%), lo que implica una muy ata participación e implicación en las tareas de aula planificadas.

Hemos querido acercarnos a las características de este grupo informante, solicitando información relativa a elementos sociodemográficos de identificación.

Tabla 1.

Sexo del alumnado

\begin{tabular}{lcc}
\hline Sexo & f & \% \\
\hline Mujer & 45 & 80.4 \\
\hline Hombre & 11 & 19.6 \\
\hline Total & 56 & 100.0 \\
\hline
\end{tabular}

El alumnado que cursa estos estudios es eminentemente femenino, al ser la presencia de las mujeres en el aula de un $80.4 \%$ (ver tabla 1 ). La edad media de este grupo es de 22 años (D.T.=3.027), con una edad mínima de 19 y una edad máxima de 32.

Tabla 2.

Formación inicial previa a la entrada en la universidad.

\begin{tabular}{lrc}
\hline Formación inicial & f & \% \\
\hline Bachillerato & 33 & 61.1 \\
\hline Ciclo Formativo Integración Social & 17 & 31.5 \\
\hline Ciclo Formativo Animación Sociocultural & 2 & 3.6 \\
\hline Ciclo Formativo TAFAD & 1 & 1.9 \\
\hline Ciclo Formativo Administración y Finanzas & 1 & 1.9 \\
\hline Total & 54 & 100.0 \\
\hline
\end{tabular}

Los datos de la tabla 2 muestran que, en su mayoría (61.1\%), el alumnado ha accedido a estos estudios por la vía del Bachillerato, aunque es relevante destacar que el 39.9\% restante ha accedido a través de un ciclo formativo de grado superior, entre los que se posiciona como más significativo el de Integración Social (31.5\%).

Tabla 3.

Ocupación habitual.

\begin{tabular}{lcc}
\hline Ocupación habitual & f & \% \\
\hline Estudio y trabajo & 18 & 32.1 \\
\hline Solo estudio & 38 & 67.9 \\
\hline Total & 56 & 100.0 \\
\hline
\end{tabular}

Es interesante destacar que el $67.9 \%$ de este grupo de estudiantes compagina estudios y trabajo durante el periodo de formación (ver tabla 3) asma como que posee experiencia en voluntariado (ver tabla 4). 
Tabla 4.

Experiencia en voluntariado.

\begin{tabular}{lcc}
\hline $\begin{array}{l}\text { Experiencia en } \\
\text { voluntariado }\end{array}$ & $\mathbf{f}$ & $\%$ \\
\hline Sí & 38 & 67.9 \\
\hline No & 18 & 32.1 \\
\hline Total & 56 & 100.0 \\
\hline
\end{tabular}

En este momento hay que señalar que han sido doce los grupos configurados para llevar a cabo las tareas prácticas demandas y, por lo tanto, doce los escenarios de intervención socioeducativa en los que ha trabajado este alumnado, siendo las y los destinatarios de la intervención profesional los siguientes: población de procedencia inmigrada, mujeres que ejercen la prostitución, menores, personas sin hogar, personas mayores, personas con autismo, mujeres gitanas y personas transexuales.

\subsection{Valoración de la propuesta por parte del alumnado}

Terminado el proceso de implementación de la propuesta de aula, este grupo de estudiantes cumplimentó, tal y como se ha informado anteriormente, un protocolo de valoración escalar dimensionado en cinco apartados que cubren la demanda de estudio: contenidos del programa práctico, actividades, escenarios socioeducativos, desarrollo del programa y satisfacción.

En un primer momento, se solicita al alumnado el cuestionamiento de diferentes elementos relacionados con los contenidos del programa práctico. Los resultados de la tabla 5 muestran un ajuste de los mismos a la práctica profesional y adaptados a las exigencias de la orientación pedagógica del equipo docente. En todo momento, los módulos teóricos han servido de acompañamiento a esta propuesta de aula y su ordenación cronológica ha sido valorada como adecuada.

Tabla 5.

Contenidos del programa práctico.

\begin{tabular}{lrrr}
\hline Contenidos del programa práctico & Media & D.T. & N \\
\hline $\begin{array}{l}\text { Se ajustan a las exigencias de las orientaciones pedagógicas del } \\
\text { profesorado }\end{array}$ & 4.14 & .773 & 56 \\
\hline $\begin{array}{l}\text { La ordenación de los contenidos es la conveniente } \\
\text { Se adecúan a los módulos teóricos de la asignatura }\end{array}$ & 4.00 & .831 & 56 \\
\hline El enfoque del programa práctico está orientado a la práctica profesional & 4.04 & .830 & 56 \\
\hline
\end{tabular}

En un segundo momento, se ha solicitado la valoración de las diferentes actividades que configuran el plan de trabajo diseñado y llevado a cabo. En este sentido, la tabla 6 muestra datos realmente positivos, al indicar que implican competencias básicas como la comprensión, el análisis, la síntesis y la evaluación. Asimismo, están diseñadas para potenciar la autonomía del alumnado y, en todo momento, se adecúan a las metas programadas. Desde la autocrítica y la autoevaluación, este alumnado señala que ha dedicado el tiempo suficiente para el desarrollo del trabajo más allá de las sesiones prácticas programadas en el horario y que, en estas, ha aprovechado la oportunidad de negociar y ser atendido por el profesorado para resolver las exigencias previstas. 
Tabla 6.

Actividades.

\begin{tabular}{lccc}
\hline Actividades & Media & D.T. & N \\
\hline Se adecúan a las exigencias de los objetivos previstos & 4.25 & .720 & 56 \\
\hline $\begin{array}{l}\text { Exigen actividad autónoma por parte del alumno } \\
\text { Implican procesos de comprensión, análisis, síntesis y evaluación }\end{array}$ & 4.34 & .880 & 56 \\
\hline $\begin{array}{l}\text { Se ha estimado convenientemente el tiempo necesario para desarrollar } \\
\text { cada módulo del trabajo }\end{array}$ & 3.43 & 1.142 & 56 \\
\hline $\begin{array}{l}\text { El tiempo estimado para cada una de las situaciones de trabajo es el } \\
\text { adecuado }\end{array}$ & 3.38 & 1.019 & 56 \\
$\begin{array}{l}\text { He dedicado el tiempo suficiente para el desarrollo del trabajo fuera del } \\
\text { aula }\end{array}$ & 4.27 & .884 & 56 \\
$\begin{array}{l}\text { He aprovechado los módulos prácticos para negociar y atender las } \\
\text { demandas del trabajo }\end{array}$ & 4.23 & .874 & 56 \\
$\begin{array}{l}\text { Se orienta al alumno hacia la utilización de los libros de consulta, } \\
\text { manuales, revistas, etc. }\end{array}$ & 3.50 & 1.044 & 56
\end{tabular}

Cuando se trata de valorar el trabajo realizado en los escenarios de intervención socioeducativa que han colaborado de una manera desinteresada y activa con los distintos grupos de estudiantes, hay que señalar que el alumnado considera que estos han respondido de manera positiva a sus necesidades y se les ha facilitado, en todo momento, llevar a cabo su propuesta de investigación e intervención (ver tabla 7).

Tabla 7.

Escenarios socioeducativos.

\begin{tabular}{lccc}
\hline Escenarios socioeducativos & Media & D.T. & N \\
$\begin{array}{l}\text { Los escenarios socioeducativos nos han posibilitado plantear } \\
\text { nuestro proceso de investigación e intervención }\end{array}$ & 4.04 & .830 & 56 \\
$\begin{array}{l}\text { El escenario, objeto de investigación e intervención, ha respondido } \\
\text { a nuestras necesidades formativas }\end{array}$ & 4.00 & .831 & 56 \\
\hline
\end{tabular}

Durante el desarrollo del programa, este alumnado considera que la implicación del profesorado, en el que se incluye la participación del alumnado mentor nombrado anteriormente, el desarrollo y seguimiento del trabajo ha sido la adecuada, así como el seguimiento realizado por las personas de referencia en los diferentes escenarios de trabajo (ver tabla 8).

Tabla 8.

Desarrollo del programa.

\begin{tabular}{lccc}
\hline Escenarios socioeducativos & Media & D.T. & N \\
\hline $\begin{array}{l}\text { Los ritmos de trabajo han sido los adecuados } \\
\begin{array}{l}\text { La participación e implicación del profesorado en el desarrollo y el } \\
\text { seguimiento del trabajo ha sido la adecuada }\end{array}\end{array}$ & 3.46 & .990 & 56 \\
$\begin{array}{l}\text { Los y las personas de referencia en los escenarios de nuestro trabajo } \\
\text { práctico nos han facilitado nuestra incorporación a los mismos }\end{array}$ & 3.96 & .786 & 56 \\
\hline
\end{tabular}

Finalmente, nos hemos querido acercar a la valoración de la satisfacción de este alumnado con respecto a su implicación, motivación y expectativas con las tareas realizadas. Puede comprobarse, por lo datos expuestos en la tabla 9, que su implicación ha sido muy activa durante las fases de desarrollo del proyecto de investigación e intervención, resultando de una gran utilidad el conocimiento de escenarios profesionales en los que en el futuro pueden ejercer su trabajo. Asimismo, les ha resultado una experiencia de gran interés siendo entendida como provechosa para el 
desarrollo de competencias profesionales. En definitiva, sienten una muy alta valoración con su participación en este tipo de propuestas de aula que combinan elementos didácticos y profesionales.

Tabla 9.

Satisfacción.

\begin{tabular}{|c|c|c|c|}
\hline Satisfacción & Media & D.T. & $\mathbf{N}$ \\
\hline Me he implicado en la propuesta práctica de las asignaturas & 4.54 & .687 & 56 \\
\hline Se han cumplido mis expectativas respecto al trabajo & 4.09 & 1.066 & 56 \\
\hline $\begin{array}{l}\text { Esta propuesta práctica es de utilidad para conocer los espacios } \\
\text { socioeducativos de nuestra realidad profesional }\end{array}$ & 4.46 & .852 & 56 \\
\hline Me ha resultado muy interesante participar en esta propuesta de trabajo & 4.32 & .897 & 56 \\
\hline $\begin{array}{l}\text { Considero que el buen ambiente de trabajo nos ha posibilitado } \\
\text { implicarnos e interesarnos por nuestra formación }\end{array}$ & 4 & .788 & 56 \\
\hline $\begin{array}{l}\text { Considero que el trabajo realizado será provechoso para mi futuro } \\
\text { profesional }\end{array}$ & 4.34 & .793 & 56 \\
\hline $\begin{array}{l}\text { Me siento satisfecha o satisfecho con mi participación en esta propuesta } \\
\text { práctica }\end{array}$ & 4.48 & .713 & 56 \\
\hline
\end{tabular}

\subsection{Necesidades de asesoramiento}

Siguiendo con las fases del estudio establecidas en el apartado metodológico de este artículo, hemos dado respuesta a la meta de recoger información sobre las necesidades de asesoramiento personal, académico y profesional de este alumnado, con un instrumento de elaboración propia diseñado con el apoyo de un profesional del servicio de inspección educativa de la Junta de Andalucía y especialista en orientación educativa. Esta valoración pretende sentar las bases para el diseño de una acción tutorial personalizada para los siguientes cursos académicos en los que se desarrolle esta experiencia didáctica.

Previo a la emisión de las demandas de asesoría, se le preguntó a este alumnado por los objetivos que deben poseer las tutorías, siendo las valoraciones emitidas muy significativas. Los datos de la tabla 10 muestran que la meta principal de esta tipología de acción docente es el acompañamiento del alumnado en su proceso educativo. En un segundo lugar, destaca el aporte de estrategias para facilitar la comprensión de la información aportada en el aula. La tercera meta perseguida es favorecer la autoestima, la motivación y la autogestión de las y los estudiantes. Comprobamos que son tres, entonces, las dimensiones más valoradas y que deben poseer las tutorías: sociales, académicas y personales.

Tabla 10.

Objetivos de las tutorías.

\begin{tabular}{|c|c|c|c|}
\hline Objetivos de las tutorías & Media & D.T. & $\mathbf{N}$ \\
\hline Establecer una relación educativa personalizada & 4.08 & .870 & 39 \\
\hline Favorecer la autoestima, motivación y autogestión & 4.16 & .866 & 37 \\
\hline $\begin{array}{l}\text { Acompañar al alumnado en su proceso educativo (planificación, mejora } \\
\text { del rendimiento...) }\end{array}$ & 4.49 & .644 & 39 \\
\hline Detectar y prevenir situaciones de abandono de los estudios & 3.90 & .995 & 39 \\
\hline $\begin{array}{l}\text { Detectar dificultades académicas que pueda tener el alumnado y derivar } \\
\text { al servicio de atención a la diversidad }\end{array}$ & 4.15 & .812 & 39 \\
\hline $\begin{array}{l}\text { Facilitar la comprensión de la información aportada en las sesiones de } \\
\text { clase }\end{array}$ & 26 & .818 & 39 \\
\hline Desarrollar habilidades y destrezas para el desarrollo & 4.10 & .718 & 39 \\
\hline Ofrecer información de las salidas profesionales de la & 4.00 & .858 & 39 \\
\hline
\end{tabular}


Atendiendo a las demandas de asesoramiento en la dimensión personal, este grupo de estudiantes considera como prioritarias el trabajo en habilidades para una comunicación efectiva, comportamiento prosocial y cooperación, asertividad y habilidades sociales básicas (ver tabla 11).

Tabla 11.

Necesidades de asesoramiento en la dimensión personal.

\begin{tabular}{lccc}
\hline Dimensión personal & Media & D.T. & $\mathbf{N}$ \\
\hline Autoestima & 3.69 & .922 & 39 \\
\hline Habilidades sociales básicas & 3.90 & .680 & 39 \\
\hline Habilidades para la comunicación efectiva & 4.00 & .827 & 39 \\
\hline Comportamiento prosocial y cooperación & 4.00 & .889 & 39 \\
\hline Asertividad & 3.90 & .788 & 39 \\
\hline Prevención y regulación de conflictos & 3.79 & .894 & 39 \\
\hline Gestión emocional & 3.74 & .880 & 39 \\
\hline
\end{tabular}

Dentro de la dimensión académica, los datos de la tabla 12 muestran que las principales necesidades detectadas hacen referencia al acompañamiento en el desarrollo del proyecto e investigación e intervención propuesto por las materias que constituyen esta acción didáctica, habilidades para el trabajo en equipo y revisión de la evaluación, haciendo referencia a la clarificación de criterios y procedimientos.

Tabla 12.

Necesidades de asesoramiento en la dimensión académica.

\begin{tabular}{lccc}
\hline Dimensión académica & Media & D.T. & N \\
\hline Materiales didácticos para el estudio & 3.69 & .766 & 39 \\
\hline Criterios de evaluación y calificación & 3.97 & .668 & 39 \\
\hline Revisión de la evaluación & 4.11 & .649 & 38 \\
\hline Habilidades para el trabajo en equipo & 4.15 & .670 & 39 \\
\hline Adquisición de hábitos de estudio & 3.54 & .884 & 39 \\
\hline Asesoramiento en la toma de decisiones respecto al desarrollo académico & 3.74 & .785 & 39 \\
\hline Acompañamiento en el desarrollo del proyecto de trabajo de INCOIN & 4.37 & .714 & 38 \\
\hline Información sobre curso de especialización y perfeccionamiento & 3.79 & .951 & 39 \\
\hline
\end{tabular}

Por último, en la dimensión profesional, las necesidades de asesoramiento que se demandan en mayor proporción son aquellas que solicitan información sobre prácticas laborales, el desarrollo de competencias para el ejercicio profesional y la aproximación al contexto laboral relacionado con la titulación (ver tabla 13).

Tabla 13

Necesidades de asesoramiento en la dimensión profesional.

\begin{tabular}{|c|c|c|c|}
\hline Dimensión profesional & Media & D.T. & $\mathbf{N}$ \\
\hline $\begin{array}{l}\text { Aproximación al contexto laboral y profesional relacionado con la } \\
\text { titulación }\end{array}$ & 4.03 & .843 & 39 \\
\hline Conocimiento del tejido asociativo de la ciudad de Córdoba & 3.85 & .875 & 39 \\
\hline Participación activa en redes de colaboración ciudadana & 3.82 & .914 & 39 \\
\hline $\begin{array}{l}\text { esarrollo de competencias profesionales a través de la realización de un } \\
\text { royecto de investigación e intervención }\end{array}$ & 4.15 & .709 & 39 \\
\hline Información sobre prácticas laborales & 4.15 & .812 & 39 \\
\hline
\end{tabular}




\section{CONCLUSIONES}

Los resultados conseguidos con la aplicación de esta propuesta de aula, entendida como innovadora, pragmática y con un alto poder de transferencia profesional, han puesto de manifiesto las siguientes conclusiones:

- Se han sentado las coordenadas que han servido para integrar los créditos prácticos de dos materias concretas del segundo curso del Grado en Educación Social que han guiado el desarrollo de una única propuesta de trabajo conjunto, de carácter integral, y concebido como una experiencia de transición de la formación académica a la práctica profesional. De este modo, y una vez analizado el impacto de esta experiencia en el alumnado y valorada por el profesorado, se va a invitar en el curso siguiente a nuevas asignaturas a incorporarse en esta dinámica de trabajo.

- El alumnado ha adquirido una serie de competencias, no solo de carácter disciplinario y curricular asociadas a las materias implicadas en el proyecto, sino una serie de habilidades y destrezas propias para el ejercicio de la profesión para la que capacita el Grado en Educación Social: autoconocimiento e inteligencia emocional, estrategias de expresión y comunicación, capacidad de liderazgo, habilidades para trabajar en equipo, estrategias para la regulación de conflictos y procedimientos para la toma de decisiones.

- El profesorado implicado en el proyecto ha dispuesto de un espacio para la reflexión conjunta y compartida de lo que supone la docencia en la universidad y la relación de la formación con la práctica profesional (contenidos, actividades, metodologías de aula, herramientas de evaluación, recursos didácticos de apoyo a la docencia, etc.). Igualmente, ha reflexionado acerca del valor añadido de la formación universitaria para el alumnado (adquisición de habilidades sociales, formación ética y democrática, valoración de la formación cultural, etc.).

- Las tutorías son entendidas por el alumnado como una estrategia facilitadora de su proceso de aprendizaje, donde se le da prioridad a la adquisición de competencias de carácter personal y profesional. Se trata de un acompañamiento efectivo que le permite al alumnado cubrir con garantías de éxito las demandas asociadas a cada una de las tareas que configuran el proyecto de investigación e intervención objeto de este trabajo.

- Los elementos que servirán para configurar en próximos cursos académicos un plan de acompañamiento tutorial son los siguientes: formación en habilidades sociales y comunicativas, asesoramiento en el desarrollo de estrategias de trabajo en equipo, planificación temporal de las tareas asociadas al proyecto de investigación e intervención, información básica sobre la evaluación de la propuesta de trabajo, información sobre prácticas laborales y formulación de las competencias profesionales asociadas a su actividad laboral.

- La mentoría entre iguales se ha mostrado como una buena estrategia para promover conjuntamente competencias generales y específicas en un contexto de aprendizaje colaborativo y dentro de un clima de confianza. 


\section{REFERENCIAS}

Arnáiz Sánchez, P., Martínez Abellán, R. \& López Castaño, L. (2000). Atención a la diversidad en contextos inclusivos. Polibea, 55, 35-58.

Álvarez González, M. (2008). La tutoría en el Espacio Europeo de Educación Superior. Revista Interuniversitaria de Formación del Profesorado, 22 (1), 4970.

Fernández-Sainero, C. (2014). La tutoría universitaria en el escenario del Espacio Europeo de Educación Superior: perfiles actuales. Teoría de la Educación. Revista Interuniversitaria, 26 (1), 161-186. DOI: http://dx.doi.org/10.14201/teoredu2014261161186

González López \& León Huertas, C. de (Coords.) (2015). Investigando en entornos diversos. De la formación a la acción. Saarbrücken (Alemania): Editorial Académica Española.

Ruiz Corbella, M., Martín Cuadrado, A. M. \& Cano Ramos, M. A. (2015). La consolidación del perfil profesional de la educación social. Respuesta al derecho para la ciudadanía. Perfiles Educativos, 37 (148), 12-19. Recuperado

de http://www.scielo.org.mx/pdf/peredu/v37n148/v37n148a17.pdf.

Tashakkori, A. \& Teddlie, Ch. (2003). Handbook of mixed methods in social and behavioral research. Londres: Sage. 